\title{
Stability of Exact and Discrete Energy for Non-Fickian Reaction-Diffusion Equations with a Variable Delay
}

\author{
Dongfang Li, ${ }^{1}$ Chao Tong, ${ }^{2,3}$ and Jinming Wen ${ }^{4}$ \\ ${ }^{1}$ School of Mathematics and Statistics, Huazhong University of Science and Technology, Wuhan 430074, China \\ ${ }^{2}$ School of Computer Science and Engineering, Beihang University, Beijing 100191, China \\ ${ }^{3}$ School of Computer Science, McGill University, Montreal, QC, Canada H3A 2K6 \\ ${ }^{4}$ Department of Mathematics and Statistics, McGill University, Montreal, QC, Canada H3A 2K6
}

Correspondence should be addressed to Jinming Wen; jinming.wen@mail.mcgill.ca

Received 4 December 2013; Revised 28 December 2013; Accepted 11 January 2014; Published 5 March 2014

Academic Editor: Adem Kilicman

Copyright (C) 2014 Dongfang Li et al. This is an open access article distributed under the Creative Commons Attribution License, which permits unrestricted use, distribution, and reproduction in any medium, provided the original work is properly cited.

\begin{abstract}
This paper is concerned with the stability of non-Fickian reaction-diffusion equations with a variable delay. It is shown that the perturbation of the energy function of the continuous problems decays exponentially, which provides a more accurate and convenient way to express the rate of decay of energy. Then, we prove that the proposed numerical methods are sufficient to preserve energy stability of the continuous problems. We end the paper with some numerical experiments on a biological model to confirm the theoretical results.
\end{abstract}

\section{Introduction}

Reaction-diffusion equations with delay are widely proposed as models for biological, physical, and control systems [15]. Over the past several years, such equations have been extensively investigated and several important properties such as existence and stability of the travelling wavefront are well understood (e.g., [6-9]). It is known that traveling waves describe transition processes from the physical point of view. If the reaction is very fast, the speed of propagation will become rather large (e.g., [10-12]). This pathologic behavior is not presented in the physical phenomena but introduced by some mathematical models (cf. [13]). In order to avoid the unphysical behavior, certain memory effects are taken into account in the mathematical models. In [13-15], the time memory flux is proposed by assuming that a flux observed at some time should have something to do with the gradient of the solution at some past time; that is,

$$
J(x, t+\delta)=-D_{2} \frac{\partial u}{\partial x}(x, t) .
$$

The first order approximation of the above formula gives

$$
J(x, t)+\delta \frac{\partial J}{\partial t}(x, t)=-D_{2} \frac{\partial u}{\partial x}(x, t) .
$$

Its solution satisfies

$$
J(x, t)=-\frac{D_{2}}{\delta} \int_{0}^{t} e^{-(t-s) / \delta} \frac{\partial u}{\partial x}(x, s) d s .
$$

In fact, the memory term is presented to avoid the infinite propagation speed in flux definition. These ideas yield the study on the following non-Fickian reaction-diffusion equation with a constant delay:

$$
\begin{aligned}
\frac{\partial u}{\partial t}(x, t)= & D_{1} \frac{\partial^{2} u}{\partial x^{2}}(x, t)+\frac{D_{2}}{\delta} \int_{0}^{t} e^{-(t-s) / \delta} \frac{\partial^{2} u}{\partial x^{2}}(x, s) d s \\
& +f(t, u(x, t), u(x, t-\tau)),
\end{aligned}
$$

where $D_{2}>0$ and $\delta>0$.

Recently, many researchers have investigated these kinds of equations. The existence and uniqueness of the solutions were derived by Chang [16]. The well posedness of the integrodifferential models was studied by Araújo et al. in [17]. Later, they further studied the effect of the integrodifferential term in the qualitative behavior of the solution of the diffusion equations in [18]. Zhang and Vandewalle [19] studied the general linear methods for solving the nonlinear integrodifferential equations with memory. Wang 
et al. investigated the asymptotic stability of exact and discrete solutions of nonlinear integrodifferential equation in [2022]. Wen et al. considered dissipativity of the functional differential equations [23]. For a more detailed description of this subject as well as its open problems, we refer the readers to the books [1-3, 24, 25], the papers [26-32], and the references therein.

In particular, some continuous and discrete energy estimates, which are determined by the $L^{2}$-norm of the solution and the estimates for the $L^{2}$-norm of the past in time of its gradient, were derived by Branco et al. in [13]. However, the derived energy estimates rely on time. When time goes to infinity, the energy estimates seem to become boundless. Later, inspired by their instructive work, Li et al. further derived some asymptotic stability of problem (1.1) in [33]. The stability result implies that the perturbation of the energy estimate tends to zero as $t$ approaches infinity. However, it does not imply anything about how long it takes to decay. Moreover, when referring to the impact of various factors (e.g., environment, temperature, and other potential effects) in some real-world problems, the model should be modified to an equation with a variable delay, which makes the numerical simulation and its analysis more complicated. Hence, some further stability results for the non-Fickian equations with a variable delay are much more significant and challenging from both the physical and mathematical points of view.

One aim of the paper is to study the exponential stability of some more general models. Consider the non-Fickian reaction-diffusion equation with a variable delay

$$
\begin{aligned}
\frac{\partial u}{\partial t}(x, t)= & D_{1} \frac{\partial^{2} u}{\partial x^{2}}(x, t)+\frac{D_{2}}{\delta} \int_{0}^{t} e^{-(t-s) / \delta} \frac{\partial^{2} u}{\partial x^{2}}(x, s) d s \\
& +f(t, u(x, t), u(x, t-\tau(t))),
\end{aligned}
$$

where $(x, t) \in\left(x_{a}, x_{b}\right) \times(0,+\infty), 0 \leq \tau(t) \leq \tau^{*}$, the boundary conditions

$$
u\left(x_{a}, t\right)=u\left(x_{b}, t\right)=0, \quad t>0
$$

and initial condition

$$
u(x, t)=u_{0}(x, t), \quad x \in\left(x_{a}, x_{b}\right), t \in\left[-\tau^{*}, 0\right] .
$$

We find that, the perturbation of the energy estimate decays exponentially. This exponential stability result provides a more accurate and convenient way to express the rate of decay. Meanwhile, the stability result can also be applied to the equations investigated in [13-15]. The given results imply that the perturbations of the energy of the equations are controlled by the initial perturbations from the systems.

The other aim is to investigate the discrete energy stability of (5). Since finite element method can be easily designed for high order of accuracy in space (see, e.g., [34, 35]), we introduce the method to solve the equation. Exponential stability of the semidiscrete system is derived. Then, the implicit Euler method is further applied to discretize the equation. And the integral is approximated by the right rectangular rule and the delay term is approximated by a linear interpolation. It is proved that the proposed numerical methods are sufficient to preserve stability of the underlying systems. Besides, if we discretize the diffusion term by using the centered difference operator, the energy stability of full discrete non-Fickian equation with a variable delay can also be derived. The given result indicates that the perturbations of the numerical solutions vanish eventually. Finally, a numerical simulation on a biological model is presented to confirm the theoretical and numerical results.

The rest of the paper is organized as follows. In Section 2, we discuss energy stability of non-Fickian delay reactiondiffusion equations. Section 3 describes in detail the stability of the numerical methods for the equation. Section 4 shows experimental studies for verifying the proposed results. Finally, conclusions and discussions for this paper are summarized in Section 5.

\section{Exponential Stability}

The following lemma can be derived from Lemma 2.1 in [36], where it was used to investigate the dissipativity of delay differential equations. Here it will play a key role in discussing the energy stability of the problem (5).

\section{Lemma 1. Suppose that}

$$
Y^{\prime}(t) \leq-\alpha(t) Y(t)+\beta(t) \sup _{t-\tau \leq \xi \leq t} Y(\xi), \quad t \geq t_{0} .
$$

Here $\tau \geq 0$ and $\alpha(t), \beta(t)$ are continuous functions such that $\alpha(t) \geq \alpha_{0}>0$, and $0 \leq \beta(t) \leq q \alpha(t)$ for all $t \geq t_{0}$ with constants $\alpha_{0}>0$ and $0 \leq q<1$. Then

$$
Y(t) \leq G \exp \left(-\mu^{*}\left(t-t_{0}\right)\right), \quad t \geq t_{0},
$$

where $G=\sup _{t_{0}-\tau \leq t \leq t_{0}}|Y(t)|$ and $\mu^{*}>0$ is defined as

$$
\mu^{*}=\inf _{t \geq t_{0}}\left\{\mu(t): \mu(t)-\alpha(t)+\beta(t) e^{\mu(t) \tau}=0\right\} .
$$

For the stability analysis, we need to consider the perturbed problem, that is, non-Fickian delay reaction-diffusion equation (5) with a different initial condition $\widetilde{u}(x, t)=$ $\tilde{u}_{0}(x, t)$. Its solution is denoted by $\tilde{u}(x, t)$ and satisfies the following equation:

$$
\begin{aligned}
\frac{\partial \widetilde{u}}{\partial t}(x, t)= & D_{1} \frac{\partial^{2} \widetilde{u}}{\partial x^{2}}(x, t)+\frac{D_{2}}{\delta} \int_{0}^{t} e^{-(t-s) / \delta} \frac{\partial^{2} \widetilde{u}}{\partial x^{2}}(x, s) d s \\
& +f(t, \tilde{u}(x, t), \tilde{u}(x, t-\tau(t))), \quad t>0, \\
\widetilde{u}(x, t)= & \widetilde{u}_{0}(x, t), \quad(x, t) \in\left(x_{a}, x_{b}\right) \times\left[-\tau^{*}, 0\right], \\
& \widetilde{u}\left(x_{a}, t\right)=\widetilde{u}\left(x_{b}, t\right)=0, \quad t>0 .
\end{aligned}
$$

Besides, let $\langle\cdot, \cdot\rangle$ denote the inner product in $L^{2}\left(x_{a}, x_{b}\right)$ and by $\|\cdot\|$ we denote the corresponding induced norm. To simplify the notation, we represent $v(\cdot, t)$ by $v(t)$ if $v$ is defined in $\left[x_{a}, x_{b}\right] \times[-\tau,+\infty)$. The energy function is defined by

$$
E_{v}(t)=\|v(t)\|^{2}+\frac{D_{2}}{\delta}\left\|\int_{0}^{t} e^{-(t-s) / \delta} \frac{\partial v}{\partial x}(s) d s\right\|^{2}
$$

Then, we have the following result. 
Theorem 2. Assume that for each $t \in[0,+\infty)$

$$
\begin{aligned}
\frac{\partial u}{\partial x}(t), \quad & \int_{0}^{t} e^{-(t-s) / \delta} \frac{\partial u}{\partial x}(s) d s, \quad \int_{0}^{t} e^{-(t-s) / \delta} \frac{\partial u_{0}}{\partial x}(s) d s, \\
& \int_{0}^{t} e^{-(t-s) / \delta} \frac{\partial \tilde{u}_{0}}{\partial x}(s) d s \in L^{2}\left(x_{a}, x_{b}\right) .
\end{aligned}
$$

If

$$
\begin{gathered}
\left\langle u_{1}-u_{2}, f\left(t, u_{1}, v_{1}\right)-f\left(t, u_{2}, v_{1}\right)\right\rangle \leq \alpha_{1}(t)\left\|u_{1}-u_{2}\right\|^{2}, \\
\left\|f\left(t, u, v_{1}\right)-f\left(t, u, v_{2}\right)\right\| \leq \beta_{1}(t)\left\|v_{1}-v_{2}\right\|,
\end{gathered}
$$

where $\alpha_{1}(t)$ and $\beta_{1}(t) \geq 0$ are continuous and there exists $0 \leq$ $q_{1}<1$ such that

$$
\begin{aligned}
q_{1} \max & \left\{-\frac{2}{\delta}, 2 \alpha_{1}(t)-\frac{2 D_{1}}{\left(x_{b}-x_{a}\right)^{2}}+\beta_{1}(t)\right\} \\
+ & \beta_{1}(t) \leq 0, \quad \text { for } t \geq 0,
\end{aligned}
$$

then

$$
E_{v}(t) \leq G_{1} \exp \left(-\mu_{1}^{*} t\right),
$$

where $G_{1}=\sup _{-\tau \leq t \leq 0}\left|E_{v}(t)\right|$ and $\mu_{1}^{*}>0$ is defined as

$$
\begin{aligned}
\mu_{1}^{*}=\inf _{t \geq t_{0}}\{\mu(t): \mu(t) \\
\quad+\max \left\{-\frac{2}{\delta}, 2 \alpha_{1}(t)-\frac{2 D_{1}}{\left(x_{b}-x_{a}\right)^{2}}+\beta_{1}(t)\right\} \\
\left.+\beta_{1}(t) e^{\mu(t) \tau}=0\right\} .
\end{aligned}
$$

Proof. Let $v(x, t)=u(x, t)-\widetilde{u}(x, t)$; we can obtain the following equation:

$$
\begin{aligned}
\frac{\partial v}{\partial t}(t)= & D_{1} \frac{\partial^{2} v}{\partial x^{2}}(t)+\frac{D_{2}}{\delta} \int_{0}^{t} e^{-(t-s) / \delta} \frac{\partial^{2} v}{\partial x^{2}}(s) d s \\
& +f(t, u(t), u(t-\tau(t))) \\
& -f(t, \tilde{u}(t), \tilde{u}(t-\tau(t))) .
\end{aligned}
$$

Multiplying each member of (18) by $v(x, t)$ with respect to $\langle\cdot, \cdot\rangle$ and integrating by part, we find

$$
\begin{aligned}
\frac{1}{2} \frac{d}{d t}\|v(t)\|^{2}= & -D_{1}\left\|\frac{\partial v}{\partial x}(t)\right\|^{2} \\
& -\frac{D_{2}}{\delta}\left\langle\int_{0}^{t} e^{-(t-s) / \delta} \frac{\partial v}{\partial x}(s) d s, \frac{\partial v}{\partial x}(t)\right\rangle \\
& +\langle f(t, u(t), u(t-\tau(t))) \\
& -f(t, \tilde{u}(t), \tilde{u}(t-\tau(t))), v(t)\rangle .
\end{aligned}
$$

As in [15],

$$
\begin{aligned}
\left\langle\frac{\partial v}{\partial x}(t), \int_{0}^{t} e^{-(t-s) / \delta} \frac{\partial v}{\partial x}(s) d s\right\rangle= & \frac{1}{2} \frac{d}{d t}\left\|\int_{0}^{t} e^{-(t-s) / \delta} \frac{\partial v}{\partial x}(s) d s\right\|^{2} \\
& +\frac{1}{\delta}\left\|\int_{0}^{t} e^{-(t-s) / \delta} \frac{\partial v}{\partial x}(s) d s\right\|^{2}
\end{aligned}
$$

we have

$$
\begin{aligned}
& \frac{d}{d t}\left(\|v(t)\|^{2}+\frac{D_{2}}{\delta}\left\|\int_{0}^{t} e^{-(t-s) / \delta} \frac{\partial v}{\partial x}(s) d s\right\|^{2}\right) \\
& =-2 D_{1}\left\|\frac{\partial v}{\partial x}(t)\right\|^{2}-\frac{2}{\delta} \frac{D_{2}}{\delta}\left\|\int_{0}^{t} e^{-(t-s) / \delta} \frac{\partial v}{\partial x}(s) d s\right\|^{2} \\
& +2\langle f(t, u(t), u(t-\tau(t))) \\
& \leq-f(t, \tilde{u}(t), \tilde{u}(t-\tau(t))), v(t)\rangle \\
& \quad-\frac{2 D_{1}}{\left(x_{b}-x_{a}\right)^{2}}\|v(t)\|^{2}-\frac{2}{\delta} \frac{D_{2}}{\delta}\left\|\int_{0}^{t} e^{-(t-s) / \delta} \frac{\partial v}{\partial x}(s) d s\right\|^{2} \\
& +2\langle f(t, u(t), u(t-\tau(t))) \\
& -f(t, \tilde{u}(t), \widetilde{u}(t-\tau(t))), v(t)\rangle,
\end{aligned}
$$

where we have used the Poincaré inequality.

Now, using assumption (14), we have

$$
\begin{aligned}
& \langle f(t, u(x, t), u(x, t-\tau(t))) \\
& -f(t, \tilde{u}(x, t), \tilde{u}(x, t-\tau(t))), v(t)\rangle \\
& =\langle f(t, u(x, t), u(x, t-\tau(t))) \\
& \quad-f(t, \tilde{u}(x, t), u(x, t-\tau(t))), v(t)\rangle \\
& \quad+\langle f(t, \tilde{u}(x, t), u(x, t-\tau(t))) \\
& \quad-f(t, \tilde{u}(x, t), \tilde{u}(x, t-\tau(t))), v(t)\rangle \\
& \leq \alpha_{1}(t)\|v(t)\|^{2}+\beta_{1}(t)\|v(t-\tau(t))\| \cdot\|v(t)\| \\
& \leq\left(\alpha_{1}(t)+\frac{\beta_{1}(t)}{2}\right)\|v(t)\|^{2}+\frac{\beta_{1}(t)}{2}\|v(t-\tau(t))\|^{2} .
\end{aligned}
$$

Therefore,

$$
\begin{aligned}
\frac{d}{d t}( & \left.\|v(t)\|^{2}+\frac{D_{2}}{\delta}\left\|\int_{0}^{t} e^{-(t-s) / \delta} \frac{\partial v}{\partial x}(s) d s\right\|^{2}\right) \\
\leq & -\frac{2}{\delta} \frac{D_{2}}{\delta}\left\|\int_{0}^{t} e^{-(t-s) / \delta} \frac{\partial v}{\partial x}(s) d s\right\|^{2} \\
& +\left(2 \alpha_{1}(t)+\beta_{1}(t)-\frac{2 D_{1}}{\left(x_{b}-x_{a}\right)^{2}}\right)\|v(t)\|^{2} \\
& +\beta_{1}(t)\|v(t-\tau(t))\|^{2}
\end{aligned}
$$




$$
\begin{aligned}
& \leq \max \left\{-\frac{2}{\delta}, 2 \alpha_{1}(t)+\beta_{1}(t)-\frac{2 D_{1}}{\left(x_{b}-x_{a}\right)^{2}}\right\} \\
& \quad \times\left(\|v(t)\|^{2}+\frac{D_{2}}{\delta}\left\|\int_{0}^{t} e^{-(t-s) / \delta} \frac{\partial v}{\partial x}(s) d s\right\|^{2}\right) \\
& +\beta_{1}(t)\|v(t-\tau(t))\|^{2} .
\end{aligned}
$$

Then, we derive the following inequality:

$$
\begin{aligned}
\frac{d}{d t} E_{v}(t) \leq & \max \left\{-\frac{2}{\delta}, 2 \alpha_{1}(t)+\beta_{1}(t)-\frac{2 D_{1}}{\left(x_{b}-x_{a}\right)^{2}}\right\} \\
& \times E_{v}(t)+\beta_{1}(t) E_{v}(t-\tau(t)) \\
\leq & \max \left\{-\frac{2}{\delta}, 2 \alpha_{1}(t)+\beta_{1}(t)-\frac{2 D_{1}}{\left(x_{b}-x_{a}\right)^{2}}\right\} \\
& \times E_{v}(t)+\beta_{1}(t) \sup _{t-\tau^{*} \leq \xi \leq t} E_{v}(\xi) .
\end{aligned}
$$
result.

Now, applying Lemma 1, we finally have the stability

It is remarkable that formula (16) implies that

$$
\|v(t)\|^{2} \leq G_{1} \exp \left(-\mu_{1}^{*} t\right) .
$$

Hence, the perturbation of the solution of the problem also decays exponentially.

When the right-hand side function of the problem (5) does not possess the delay term, the problem degenerates into a non-Fickian reaction-diffusion equation:

$$
\begin{aligned}
\frac{\partial u}{\partial t}(x, t)= & D_{1} \frac{\partial^{2} u}{\partial x^{2}}(x, t)+\frac{D_{2}}{\delta} \int_{0}^{t} e^{-(t-s) / \delta} \frac{\partial^{2} u}{\partial x^{2}}(x, s) d s \\
& +f(t, u(x, t)), \quad t>0,
\end{aligned}
$$

which is also discussed in [13]. We have the following conclusion.

Corollary 3. Assume that for each $t \in[0,+\infty)$

$$
\begin{gathered}
\frac{\partial u}{\partial x}(t), \quad \int_{0}^{t} e^{-(t-s) / \delta} \frac{\partial u}{\partial x}(s) d s \\
\int_{0}^{t} e^{-(t-s) / \delta} \frac{\partial u_{0}}{\partial x}(s) d s \in L^{2}\left(x_{a}, x_{b}\right) .
\end{gathered}
$$

If

$$
\langle f(t, u)-f(t, v), u-v\rangle \leq \alpha_{2}(t)\|u-v\|^{2},
$$

where $\alpha_{2}(t)$ are continuous and $2 \alpha_{2}(t)-2 D_{1} /\left(x_{b}-x_{a}\right)^{2}<0$, for $t \geq 0$. Then there exist constants $G_{2}$ and $\mu_{2}^{*}$ such that

$$
E_{v}(t) \leq G_{2} \exp \left(-\mu_{2}^{*} t\right)
$$

When $D_{1}=0$, the problem (5) degenerates into an integrodifferential delay equation,

$$
\begin{aligned}
\frac{\partial u}{\partial t}(x, t)= & \frac{D_{2}}{\delta} \int_{0}^{t} e^{-(t-s) / \delta} \frac{\partial^{2} u}{\partial x^{2}}(x, s) d s \\
& +f(t, u(x, t), u(x, t-\tau(t))), \quad t>0
\end{aligned}
$$

which is investigated in $[14,15]$. We have the following conclusion.

Corollary 4. Assume that for each $t \in[0,+\infty)$

$$
\begin{gathered}
\frac{\partial u}{\partial x}(t), \quad \int_{0}^{t} e^{-(t-s) / \delta} \frac{\partial u}{\partial x}(s) d s, \\
\int_{0}^{t} e^{-(t-s) / \delta} \frac{\partial u_{0}}{\partial x}(s) d s \in L^{2}\left(x_{a}, x_{b}\right) . \\
\text { If } \begin{array}{l}
\left\langle u_{1}-u_{2}, f\left(t, u_{1}, v_{1}\right)-f\left(t, u_{2}, v_{1}\right)\right\rangle \leq \alpha_{3}(t)\left\|u_{1}-u_{2}\right\|^{2}, \\
\left\|f\left(t, u, v_{1}\right)-f\left(t, u, v_{2}\right)\right\| \leq \beta_{3}(t)\left\|v_{1}-v_{2}\right\|,
\end{array}
\end{gathered}
$$

where $\alpha_{3}(t)$ and $\beta_{3}(t) \geq 0$ are continuous and there exists $0 \leq$ $q_{3}<1$ such that

$$
q_{1} \max \left\{-\frac{2}{\delta}, 2 \alpha_{1}(t)+\beta_{1}(t)\right\}+\beta_{1}(t)<0, \quad \text { for } t \geq 0,
$$

then there exist constants $G_{3}$ and $\mu_{3}^{*}$ such that

$$
E_{v}(t) \leq G_{3} \exp \left(-\mu_{3}^{*} t\right)
$$

If we assume that

$$
\langle f(t, u, v), u\rangle \leq \alpha_{1}(t)\|u\|^{2}+\beta_{1}(t)\|v\|^{2}+\gamma_{1}(t),
$$

where $\beta_{1}(t) \geq 0$ and $\gamma_{1}(t) \geq 0$ and $\alpha_{1}(t)$ are continuous functions, there may exist a bounded set $\mathscr{B}$ and a time $t^{*}$, such that for any given initial function $u_{0}(x, t),(x, t) \epsilon$ $\left[x_{a}, x_{b}\right] \times[-\tau, 0]$, the corresponding energy $\left\|E_{u}(t)\right\|$ of the problem is contained in $\mathscr{B}$ for all $t \geq t^{*}$. The analogous results and their conclusions for non-Fickian equations with a variable delay can be derived without any difficulty. We do not list them here for brevity.

\section{Stability of the Numerical Approximation}

In this section, we will concentrate on the stability of two kinds of numerical approximation. Here and below, for the discretization of system (5), we divide the interval $I=\left[x_{a}, x_{b}\right]$ with a mesh: $x_{a}=x_{0}<x_{1}<\cdots<x_{N}=x_{b}$ with the space stepsize $h=\left(x_{b}-x_{a}\right) / N$, where $N$ is a positive integer. And $k=1 / N_{k}$ is a time stepsize, where $N_{k}$ is a positive integer. We will numerically solve the problem at time $n k, n=1,2, \ldots$ 
3.1. Stability of the Finite Element Approximation. Firstly, the finite element method is introduced to discrete the problems. Let $S^{h}$ be a finite dimensional subspace of $H_{0}^{1}(I)$; then the semidiscrete finite element method is to find $U \in S^{h}$, such that, for all test functions, $\chi_{i} \in S^{h}$ and $1 \leq i \leq N$, satisfying

$$
\begin{gathered}
\left(\frac{\partial u_{h}}{\partial t}, \chi\right)+\left(D_{1} \frac{\partial u_{h}}{\partial x}+\frac{D_{2}}{\delta} \int_{0}^{t} e^{-(t-s) / \delta} \frac{\partial u_{h}}{\partial x}(s) d s, \frac{\partial \chi}{\partial x}\right) \\
=\left(f\left(t, u_{h}(x, t), u_{h}(x, t-\tau(t))\right), \chi\right) .
\end{gathered}
$$

The exponential stability of semidiscrete approximation is shown as follows.

Theorem 5. Assume that conditions in Theorem 2 hold; then the difference $E_{v_{h}}(t)$ satisfies the following formulae:

$$
\left\|E_{v_{h}}(t)\right\|^{2} \leq G_{1} \exp \left(-\mu^{*}\left(t-t_{0}\right)\right), \quad t \geq t_{0},
$$

where the parameters $G_{1}$ and $\mu^{*}$ are defined in Theorem 2 and $v_{h}=u_{h}-\widetilde{u}_{h}$ with $\widetilde{u}_{h}$ satisfying the semidiscrete finite element approximation to the perturbed problem.

Proof. The proof is similar to that of Theorem 2.

If we further apply the right rectangular rule to approximate the integral and implicit Euler method to discrete (36), then the full discrete numerical approximation is to find $u_{h}^{n} \in$ $S^{h}$, such that, for all test functions, $\chi \in S_{h}$ and $1 \leq i \leq N$, satisfying for $n=0,1,2, \ldots$

$$
\begin{aligned}
& \left(\frac{u_{h}^{n+1}-u_{h}^{n}}{k}, \chi\right)+\left(D_{1} \frac{\partial}{\partial x} u_{h}^{n+1}+\frac{D_{2} k}{\delta}\right. \\
& \left.\quad \times \sum_{i=1}^{n+1} e^{-\left(t_{n+1}-t_{i}\right) / \delta} \frac{\partial}{\partial x} u_{h}^{i}, \frac{\partial \chi}{\partial x}\right) \\
& =\left(f\left(t, u_{h}^{n+1}, \bar{u}_{h}^{n+1}\right), \chi\right) .
\end{aligned}
$$

Here $\bar{u}_{h}^{n+1}$ denotes an approximation to $u\left(x, t_{n+1}-\tau\left(t_{n+1}\right)\right)$, which is obtained by the following linear interpolation procedure at the point $t_{n+1}-\tau\left(t_{n+1}\right)$ :

$$
\bar{u}_{h}^{n+1}=\theta_{n} u_{h}^{n+1-m_{n}}+\left(1-\theta_{n}\right) u_{h}^{n-m_{n}},
$$

where $\tau(t)=\left(m_{n}-\theta_{n}\right) k$ with integer $m_{n} \geq 1$ and $\theta_{n} \in[0,1]$.

Now, we can establish the discrete version of the energy:

$$
\widetilde{E}\left(u_{h}^{n}\right)=\left\|u_{h}^{n}\right\|^{2}+e^{-2(k / \delta)} \frac{D_{2}}{\delta}\left\|k \sum_{j=1}^{n} e^{-\left(t_{n}-t_{j}\right) / \delta} \frac{\partial}{\partial x} u_{h}^{l}\right\|^{2} .
$$

Then, we have the following result.

Theorem 6. Assume that conditions (14) and (15) hold. Then there exists a constant $0 \leq q_{2}<1$ such that

$$
\widetilde{E}\left(v_{h}^{n+1}\right) \leq q_{2} \widetilde{E}\left(v_{h}^{n}\right),
$$

where $v_{h}^{n}=u_{h}^{n}-\widetilde{u}_{h}^{n}$ and $\widetilde{u}_{h}^{n}\left(x_{i}\right)$ is the full discrete approximations to the perturbed problem.
Proof. The difference $v_{h}^{n}$ satisfies the following equation:

$$
\begin{aligned}
&\left(\frac{v_{h}^{n+1}-v_{h}^{n}}{k}, \chi\right)+\left(D_{1} \frac{\partial}{\partial x} v_{h}^{n+1}+\frac{D_{2} k}{\delta}\right. \\
&\left.\times \sum_{i=1}^{n+1} e^{-\left(t_{n+1}-t_{i}\right) / \delta} \frac{\partial}{\partial x} v_{h}^{i}, \frac{\partial \chi}{\partial x}\right) \\
&=\left(f\left(t, u_{h}^{n+1}, \bar{u}_{h}^{n+1}\right)-f\left(t, \widetilde{u}_{h}^{n+1}, \overline{\widetilde{u}}_{h}^{n+1}\right), \chi\right) .
\end{aligned}
$$

Taking $\chi=v_{h}^{n+1}$ in the above formula, we find that

$$
\begin{aligned}
& \left\|v_{h}^{n+1}\right\|^{2}=\left(v_{h}^{n}, v_{h}^{n+1}\right)-k D_{1}\left\|\frac{\partial}{\partial x} v_{h}^{n+1}\right\|^{2}-\frac{D_{2} k^{2}}{\delta} \\
& \times\left(\sum_{j=1}^{n+1} e^{-\left(t_{n}-t_{j}\right) / \delta} \frac{\partial}{\partial x} v_{h}^{j}, \frac{\partial}{\partial x} v_{h}^{n+1}\right) \\
& +k\left(f\left(t_{n+1}, u_{h}^{n+1}, \bar{u}_{h}^{n+1}\right)\right. \\
& \left.-f\left(t_{n+1}, \tilde{u}_{h}^{n+1}, \overline{\tilde{u}}_{h}^{n+1}\right), v_{h}^{n+1}\right) \\
& =\left(v_{h}^{n}, v_{h}^{n+1}\right)-k D_{1}\left\|\frac{\partial}{\partial x} v_{h}^{n+1}\right\|^{2}-\frac{D_{2} k^{2}}{\delta} \\
& \times\left(\sum_{j=1}^{n+1} e^{-\left(t_{n}-t_{j}\right) / \delta} \frac{\partial}{\partial x} v_{h}^{j}, \frac{\partial}{\partial x} v_{h}^{n+1}\right) \\
& +k\left(f\left(t_{n+1}, u_{h}^{n+1}, \bar{u}_{h}^{n+1}\right)\right. \\
& \left.-f\left(t_{n+1}, \widetilde{u}_{h}^{n+1}, \bar{u}_{h}^{n+1}\right), v_{h}^{n+1}\right) \\
& +k\left(f\left(t_{n+1}, \tilde{u}_{h}^{n+1}, \bar{u}_{h}^{n+1}\right)\right. \\
& \left.-f\left(t_{n+1}, \tilde{u}_{h}^{n+1}, \overline{\widetilde{u}}_{h}^{n+1}\right), v_{h}^{n+1}\right) \\
& \leq\left(v_{h}^{n}, v_{h}^{n+1}\right)-k D_{1}\left\|\frac{\partial}{\partial x} v_{h}^{n+1}\right\|^{2}-\frac{D_{2} k^{2}}{\delta} \\
& \times\left(\sum_{j=1}^{n+1} e^{-\left(t_{n}-t_{j}\right) / \delta} \frac{\partial}{\partial x} v_{h}^{j}, \frac{\partial}{\partial x} v_{h}^{n+1}\right) \\
& +\left(\alpha_{n+1}+\frac{\beta_{n+1}}{2}\right) k\left\|v_{h}^{n+1}\right\|^{2}+\frac{\beta_{n+1}}{2} k\left\|\bar{v}_{h}^{n+1}\right\|^{2},
\end{aligned}
$$

where we have used condition (14).

As in [15], we have

$$
\begin{gathered}
\left(\sum_{j=1}^{n+1} e^{-\left(t_{n+1}-t_{j}\right) / \delta} \frac{\partial}{\partial x} v_{h}^{j}, \frac{\partial}{\partial x} v_{h}^{n+1}\right) \\
=\frac{1}{2}\left\|\sum_{l=1}^{n+1} e^{-\left(t_{n+1}-t_{l}\right) / \delta} \frac{\partial}{\partial x} v_{h}^{l}\right\|^{2}
\end{gathered}
$$




$$
\begin{aligned}
& -\frac{1}{2} e^{-2(k / \delta)}\left\|\sum_{l=1}^{n} e^{-\left(t_{n}-t_{l}\right) / \delta} \frac{\partial}{\partial x} v_{h}^{l}\right\|^{2} \\
& +\frac{1}{2}\left\|\frac{\partial}{\partial x} v_{h}^{n+1}\right\|^{2} .
\end{aligned}
$$

Substituting (44) into (43) yields

$$
\begin{aligned}
\left\|v_{h}^{n+1}\right\|^{2}+\frac{D_{2}}{2 \delta}\left\|k \sum_{l=1}^{n+1} e^{-\left(t_{n+1}-t_{l}\right) / \delta} \frac{\partial}{\partial x} v_{h}^{l}\right\|^{2} \\
=\left(v_{h}^{n}, v_{h}^{n+1}\right)-k D_{1}\left\|\frac{\partial}{\partial x} v_{h}^{n+1}\right\|^{2}+\frac{D_{2}}{2 \delta} e^{-2(k / \delta)} \\
\quad \times\left\|k \sum_{l=1}^{n} e^{-\left(t_{n}-t_{l}\right) / \delta} \frac{\partial}{\partial x} v_{h}^{l}\right\|^{2}-\frac{D_{2} k^{2}}{2 \delta}\left\|\frac{\partial}{\partial x} v_{h}^{n+1}\right\|^{2} \\
+\left(\alpha_{n+1}+\frac{\beta_{n+1}}{2}\right) k\left\|v_{h}^{n+1}\right\|^{2}+\frac{\beta_{n+1}}{2} k\left\|\bar{v}_{h}^{n+1}\right\|^{2} .
\end{aligned}
$$

Applying the following discrete Poincaré inequality

$$
-\left\|\frac{\partial}{\partial x} v_{h}^{n+1}\right\|^{2} \leq-\frac{1}{\left(x_{b}-x_{a}\right)^{2}}\left\|v_{h}^{n+1}\right\|^{2}
$$

and $\left(v_{h}^{n}, v_{h}^{n+1}\right) \leq(1 / 2)\left\|v_{h}^{n+1}\right\|^{2}+(1 / 2)\left\|v_{h}^{n}\right\|^{2}$ to $(45)$, we arrive at

$$
\begin{aligned}
(1- & \left.2 k \alpha_{n+1}-k \beta_{n+1}+\frac{2 k D_{1}}{\left(x_{b}-x_{a}\right)^{2}}\right)\left\|v_{h}^{n+1}\right\|^{2} \\
& +\frac{D_{2}}{\delta}\left\|k \sum_{l=1}^{n+1} e^{-\left(t_{n+1}-t_{l}\right) / \delta} \frac{\partial}{\partial x} v_{h}^{l}\right\|^{2} \\
\leq & \left\|v_{h}^{n}\right\|^{2}+e^{-2(k / \delta)} \frac{D_{2}}{\delta}\left\|k \sum_{l=1}^{n} e^{-\left(t_{n}-t_{l}\right) / \delta} \frac{\partial}{\partial x} v_{h}^{l}\right\|^{2} \\
& +k \beta_{n+1}\left\|\bar{v}_{h}^{n+1}\right\|^{2} \\
= & \left\|v_{h}^{n}\right\|^{2}+e^{-2(k / \delta)} \frac{D_{2}}{\delta}\left\|k \sum_{l=1}^{n} e^{-\left(t_{n}-t_{l}\right) / \delta} \frac{\partial}{\partial x} v_{h}^{l}\right\|^{2} \\
& +k \beta_{n+1}\left\|\theta_{n} v_{n}^{n+1-m_{n}}+\left(1-\theta_{n}\right) v_{h}^{n-m_{n}}\right\|^{2} \\
= & \left\|v_{h}^{n}\right\|^{2}+e^{-2(k / \delta)} \frac{D_{2}}{\delta}\left\|k \sum_{l=1}^{n} e^{-\left(t_{n}-t_{l}\right) / \delta} \frac{\partial}{\partial x} v_{h}^{l}\right\|^{2} \\
& +k \beta_{n+1}\left(\theta_{n}^{2}\left\|v_{n}^{n+1-m_{n}}\right\|^{2}+\left(1-\theta_{n}\right)^{2}\left\|v_{h}^{n-m_{n}}\right\|^{2}\right. \\
& \left.+2 \theta_{n}\left(1-\theta_{n}\right)\left\|v_{n}^{n+1-m_{n}}\right\|\left\|v_{h}^{n-m_{n}}\right\|\right)
\end{aligned}
$$

$$
\begin{aligned}
\leq & \left\|v_{h}^{n}\right\|^{2}+e^{-2(k / \delta)} \frac{D_{2}}{\delta}\left\|k \sum_{l=1}^{n} e^{-\left(t_{n}-t_{l}\right) / \delta} \frac{\partial}{\partial x} v_{h}^{l}\right\|^{2} \\
& +k \beta_{n+1}\left(\theta_{n}\left\|v_{n}^{n+1-m_{n}}\right\|^{2}+\left(1-\theta_{n}\right)\left\|v_{h}^{n-m_{n}}\right\|^{2}\right) \\
\leq & \widetilde{E}\left(v_{h}^{n}\right)+k \beta_{n+1} \max \left\{\left\|v_{h}^{n+1-m_{n}}\right\|^{2},\left\|v_{h}^{n-m_{n}}\right\|^{2}\right\} \\
\leq & \widetilde{E}\left(v_{h}^{n}\right)+k \beta_{n+1} \max \left\{\widetilde{E}\left(v_{h}^{n+1-m_{n}}\right), \widetilde{E}\left(v_{h}^{n-m_{n}}\right)\right\} \\
\leq & \left(1+k \beta_{n+1}\right) \max \left\{\widetilde{E}\left(v_{h}^{n}\right), \widetilde{E}\left(v_{h}^{n+1-m_{n}}\right), \widetilde{E}\left(v_{h}^{n-m_{n}}\right)\right\},
\end{aligned}
$$

where we have used the inequality $2 \alpha \beta \leq \alpha^{2}+\beta^{2}$.

It follows from (47) that

$$
\begin{aligned}
\min & \left\{C_{n+1}, e^{2(k / \delta)}\right\} \widetilde{E}\left(v_{h}^{n+1}\right) \\
\leq & \left(1-2 k \alpha_{n+1}-k \beta_{n+1}+\frac{2 k D_{1}}{\left(x_{b}-x_{a}\right)^{2}}\right) \\
& \times\left\|v_{h}^{n+1}\right\|^{2}+\frac{D_{2}}{\delta}\left\|k \sum_{l=1}^{n+1} e^{-\left(t_{n+1}-t_{l}\right) / \delta} \frac{\partial}{\partial x} v_{h}^{l}\right\|^{2} \\
\leq & \left(1+k \beta_{n+1}\right) \max \left\{\widetilde{E}\left(v_{h}^{n}\right), \widetilde{E}\left(v_{h}^{n+1-m_{n}}\right), \widetilde{E}\left(v_{h}^{n-m_{n}}\right)\right\},
\end{aligned}
$$

where $C_{n+1}=\left(1-2 k \alpha_{n+1}-k \beta_{n+1}+2 k D_{1} /\left(x_{b}-x_{a}\right)^{2}\right)$.

Therefore,

$$
\begin{aligned}
\widetilde{E}\left(v_{h}^{n+1}\right) \leq & \frac{\left(1+k \beta_{n+1}\right)}{\min \left\{C_{n+1}, e^{2(k / \delta)}\right\}} \\
& \times \max \left\{\widetilde{E}\left(v_{h}^{n}\right), \widetilde{E}\left(v_{h}^{n+1-m_{n}}\right), \widetilde{E}\left(v_{h}^{n-m_{n}}\right)\right\} .
\end{aligned}
$$

In fact, in view of condition (15), there exists a constant $\varrho$, such that

$$
\begin{aligned}
& \frac{1}{C_{n+1}}+\frac{k \beta_{n+1}}{C_{n+1}} \leq \varrho<1, \\
& e^{-2(k / \delta)}\left(1+k \beta_{n+1}\right) \leq e^{-k \beta_{n+1}}\left(1+k \beta_{n+1}\right) \\
&=\left(1-k \beta_{n+1}+\mathcal{O}\left(k^{2}\right)\right) \\
& \times\left(1+k \beta_{n+1}\right) \leq \varrho<1 .
\end{aligned}
$$

Hence,

$$
\frac{1+k \beta_{n+1}}{\min \left\{C_{n+1}, e^{2(k / \delta)}\right\}} \leq \varrho<1, \quad n=1,2, \ldots
$$

Together with (49) and (52), we have the final conclusion.

Theorem 5 shows that, for any given initial perturbation, the perturbation of the corresponding discrete energy of the problem decreases in time. Meantime, noting that $\left\|k \sum_{l=1}^{n} e^{-\left(t_{n}-t_{l}\right) / \delta}(\partial / \partial x) v_{h}^{l}\right\|^{2}>0$ in the energy function, the difference $\left\|v_{h}^{n}\right\|$ tends to zero very quickly. 
3.2. Stability of the Finite Difference Approximation. In this subsection, we will apply the central finite difference method to discrete the diffusion term and discuss the stability of the full discrete problems.

Let $k$ be the time stepsize and $U_{h}^{n}\left(x_{i}\right)$ denote the numerical approximation to $U\left(x_{i}, t_{n}\right)$ with $t_{n}=n k$; then the full discrete finite difference approximation for (5) can be written as

$$
\begin{aligned}
U_{h}^{n+1}\left(x_{i}\right)= & U_{h}^{n}\left(x_{i}\right)+k D_{1} D_{2, x} U_{h}^{n+1}\left(x_{i}\right)+k^{2} \frac{D_{2}}{\delta} \\
& \times \sum_{l=1}^{n+1} e^{-\left(t_{n+1}-t_{l}\right) / \delta} D_{2, x} U_{h}^{l}\left(x_{i}\right) \\
& +k f\left(t_{n+1}, U_{h}^{n+1}\left(x_{i}\right), \bar{U}_{h}^{n+1}\left(x_{i}\right)\right), \\
n & =0,1,2, \ldots,
\end{aligned}
$$

where

$$
\begin{array}{r}
D_{2, x} U_{h}\left(x_{i}\right)=\frac{U_{h}\left(x_{i+1}\right)-2 U_{h}\left(x_{i}\right)+U_{h}\left(x_{i-1}\right)}{h^{2}}, \\
i=1, \ldots, N-1,
\end{array}
$$

and the argument $\bar{U}_{h}^{n+1}(x)$ denotes an approximation to $u\left(x, t_{n+1}-\tau\left(t_{n+1}\right)\right)$, which is obtained by the linear interpolation procedure at the point $t_{n+1}-\tau\left(t_{n+1}\right)$

$$
\bar{U}_{h}^{n+1}=\theta_{n} U_{h}^{n+1-m_{n}}+\left(1-\theta_{n}\right) U_{h}^{n-m_{n}},
$$

where $\tau(t)=\left(m_{n}-\theta_{n}\right) k$ with integer $m_{n} \geq 1$ and $\theta_{n} \in[0,1]$.

The discrete version of the energy of the finite difference approximation is defined by

$$
\begin{aligned}
\widetilde{\widetilde{E}}\left(U_{h}^{n+1}\right)= & \left\|U_{h}^{n+1}\right\|_{L^{2}\left(I_{h}\right)}^{2}+e^{-2(k / \delta)} \frac{D_{2}}{\delta} \\
& \times\left\|k \sum_{l=1}^{n+1} e^{-\left(t_{n+1}-t_{l}\right) / \delta} D_{-x} U_{h}^{l}\right\|_{L^{2}\left(I_{h},+\right)}^{2},
\end{aligned}
$$

where $D_{-x}$ is the backward finite difference operator:

$$
\begin{gathered}
\left\|U_{h}\right\|_{L^{2}\left(I_{h}\right)}^{2}=\left(U_{h}, U_{h}\right)_{h}=\left(U_{h}, U_{h}\right)_{h}=h \sum_{i=1}^{N-1} U_{h}^{2}\left(x_{i}\right), \\
\|V\|_{L^{2}\left(I_{h},+\right)}^{2}=(V, V)_{h,+}=h \sum_{i=1}^{N} V\left(x_{i}\right) V\left(x_{i}\right), \\
V=e^{-\left(t_{n+1}-t_{l}\right) / \delta} D_{-x} U_{h}^{l} .
\end{gathered}
$$

Theorem 7. Assume that conditions (14) and (15) hold. Then there exists a constant $0 \leq q_{3}<1$ such that

$$
\widetilde{\widetilde{E}}\left(V_{h}^{n+1}\right) \leq q_{3} \widetilde{\widetilde{E}}\left(V_{h}^{n}\right)
$$

where $V_{h}^{n}=U_{h}^{n}-\widetilde{U}_{h}^{n}$ and $\widetilde{U}_{h}^{n}\left(x_{i}\right)$ is the full discrete finite difference approximations to the perturbed problem.
Proof. The proof is similar to the one done in Theorem 6 with the following relation:

$$
\begin{aligned}
& \left(\sum_{j=1}^{n+1} e^{-\left(t_{n+1}-t_{j}\right) / \delta} D_{-x} v_{h}^{j}, D_{-x} v_{h}^{n+1}\right)_{h,+} \\
& =\frac{1}{2}\left\|\sum_{l=1}^{n+1} e^{-\left(t_{n+1}-t_{l}\right) / \delta} D_{-x} v_{h}^{l}\right\|_{L^{2}\left(I_{h},+\right)}^{2} \\
& \quad-\frac{1}{2} e^{-2(k / \delta)}\left\|\sum_{l=1}^{n} e^{-\left(t_{n+1}-t_{l}\right) / \delta} D_{-x} v_{h}^{l}\right\|_{L^{2}\left(I_{h},+\right)}^{2} \\
& +\frac{1}{2}\left\|D_{-x} v_{h}^{n+1}\right\|_{L^{2}\left(I_{h},+\right)}^{2} .
\end{aligned}
$$

\section{Application}

In this section, we will provide a numerical experiment to illustrate the given results.

Consider the following Non-Fickian Mackey-Glass equations [33]:

$$
\begin{aligned}
& \frac{\partial u}{\partial t}(x, t)= D_{1} \frac{\partial^{2} u}{\partial x^{2}}(x, t)+\frac{D_{2}}{\delta} \int_{0}^{t} e^{-(t-s) / \delta} \frac{\partial^{2} u}{\partial x^{2}}(x, s) d s \\
&-a u(x, t)+\frac{b u(x, t-\tau(t))}{1+u^{m}(x, t-\tau(t))}, \\
& u\left(x_{a}, t\right)=u\left(x_{b}, t\right)=0, \quad t>0, \\
& u(x, t)=\varphi(x, t), \quad(x, t) \in[0, \pi] \times\left[-\tau^{*}, 0\right],
\end{aligned}
$$

where $a, b, D_{1}, D_{2}$, and $\delta$ are given parameters, $m$ is an even positive integer, and $\tau(t)$ is a nonnegative function.

There is no difficulty in verifying that condition (14) holds $\left(\alpha_{1}(t)=-a, \beta_{1}(t)=b\right)$. If there exists $0 \leq q_{1}<1$ such that $q_{1} \max \left\{-2 / \delta,-2 a-2 D_{1} /\left(x_{b}-x_{a}\right)^{2}+b\right\}+b<0$, the perturbation of the energy estimate decays exponentially. For the numerical simulation of the model, we set the parameter $D_{1}=D_{2}=\delta=1, m=2, \tau(t)=1-0.1|\sin (t)|$, the space stepsize $h=\pi / 10$, and time stepsize $k=0.1$ and apply 1-degree finite element method to solve the problem on $[0, \pi] \times[0,20]$ with the following different initial conditions:

$$
\begin{gathered}
u_{0}(x, t)=(2+\cos (t)) \sin (x), \\
\widetilde{u}_{0}(x, t)=10 x(\pi-x) .
\end{gathered}
$$

Some statistics of the numerical results with different parameters $a$ and $b$ are shown in Tables 1 and 2, where the discrete energy $\widetilde{E}\left(v_{h}^{n}\right)$ and $\left\|v_{h}^{n}\right\|^{2}$ of the problems decreases in time very quickly. Moreover, The numerical differences $\left|v_{h}^{n}\left(x_{i}\right)\right|$ with the parameters $a=2$ and $b=1$ are shown in Figure 1, where $x_{i}=i h$ with $i=1,2, \ldots, 9$. The numerical differences (the blue lines) are all bounded by the exponential function $y=20 \exp (-t)$ (the red line). Clearly, they all confirm the results in this paper. 
TABLE 1: The discrete energy $\widetilde{E}\left(v_{h}^{n}\right)$ at different time.

\begin{tabular}{|c|c|c|c|c|c|}
\hline$\widetilde{E}\left(v_{h}^{n}\right)$ & $t=2$ & $t=4$ & $t=6$ & $t=8$ & $t=10$ \\
\hline$a=1, b=1$ & 1.37 & $1.01 E-1$ & $9.17 E-4$ & $6.90 E-5$ & $6.53 E-6$ \\
\hline$a=2, b=1$ & $5.28 E-1$ & $2.21 E-2$ & $1.72 E-4$ & $6.32 E-6$ & $2.08 E-7$ \\
\hline$a=3, b=1$ & $2.88 E-1$ & $7.73 E-3$ & $8.20 E-5$ & $1.55 E-6$ & $3.33 E-8$ \\
\hline$a=4, b=1$ & $1.87 E-1$ & $3.94 E-3$ & $5.32 E-5$ & $8.98 E-7$ & $1.69 E-8$ \\
\hline
\end{tabular}

TABLE 2: The difference $\left\|v_{h}^{n}\right\|^{2}$ at different time.

\begin{tabular}{lccccc}
\hline$\left\|v_{h}^{n}\right\|^{2}$ & $t=2$ & $t=4$ & $t=6$ & $t=8$ & $t=10$ \\
\hline$a=1, b=1$ & $9.52 E-1$ & $8.43 E-2$ & $4.37 E-4$ & $5.80 E-5$ & $5.77 E-6$ \\
$a=2, b=1$ & $2.82 E-1$ & $4.56 E-2$ & $2.86 E-5$ & $3.53 E-6$ & $1.38 E-7$ \\
$a=3, b=1$ & $1.19 E-1$ & $4.00 E-3$ & $8.21 E-6$ & $1.92 E-7$ & $7.38 E-9$ \\
$a=4, b=1$ & $6.16 E-2$ & $1.39 E-3$ & $4.56 E-6$ & $3.40 E-9$ & $5.04 E-10$ \\
\hline
\end{tabular}

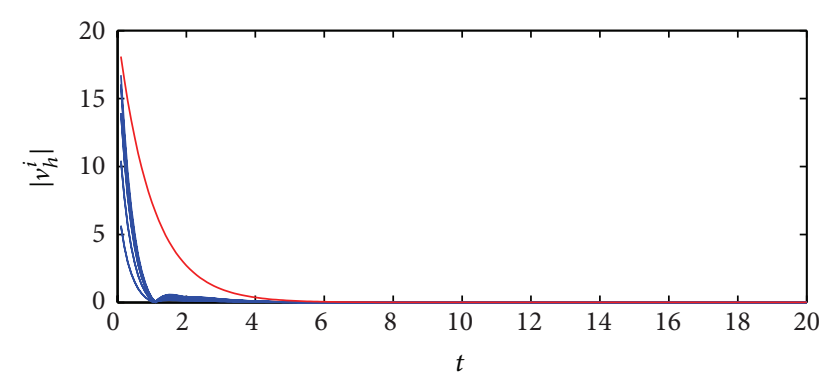

FIgURE 1: The difference $\left|v_{h}^{n}\left(x_{i}\right)\right|$ at different time.

\section{Conclusions}

In this paper, we have investigated the stability of continuous and discrete non-Fickian delay reaction-diffusion equations with a variable delay. Firstly, we show that the perturbation of the energy estimate decays exponentially. The present stability results for a more general case improved that of our previous paper. Secondly, the numerical analysis indicates that the finite element method or central finite difference method, combined with implicit Euler method and a linear interpolation procedure for the delay term, can preserve the stability of the energy function of the equations. All the above findings are confirmed by using the numerical methods on the non-Fickian Mackey-Glass equations.

\section{Conflict of Interests}

The authors declare that there is no conflict of interests regarding the publication of this paper.

\section{Acknowledgments}

This work is supported by the National Natural Science Foundation of China under Grants 11201161, 11171125, 91130003, 61170296, and 61190125; the Fundamental Research Funds for the Central Universities under Grant no. 2012QN033; the Research Fund of the State Key Laboratory of Software
Development Environment under Grant no. BUAA SKLSDE2012ZX-17; the Program for New Century Excellent Talents in University under Grant no. NECT-09-0028; and the Natural Science Foundation of Beijing, China, under Grant no. 4123101.

\section{References}

[1] R. P. Agarwal, L. Berezansky, E. Braverman, and A. Domoshnitsky, Nonoscillation Theory of Functional Differential Equations with Applications, Springer, New York, NY, USA, 2012.

[2] H. Brunner, Collocation Methods for Volterra Integral and Related Functional Differential Equations, Cambridge University Press, Cambridge, UK, 2004.

[3] J. H. Wu, Theory and Application of Partial Functional Differential Equation, vol. 119 of Applied Mathematical Sciences, Springer-Verlag, 1996.

[4] L. Berezansky, E. Braverman, and L. Idels, "Nicholson's blowflies differential equations revisited: main results and open problems," Applied Mathematical Modelling, vol. 34, no. 6, pp. 1405$1417,2010$.

[5] D. Li, C. Zhang, and H. Qin, "LDG method for reactiondiffusion dynamical systems with time delay," Applied Mathematics and Computation, vol. 217, no. 22, pp. 9173-9181, 2011.

[6] M. Mei, C.-K. Lin, C.-T. Lin, and J. W.-H. So, "Traveling wavefronts for time-delayed reaction-diffusion equation. I. Local nonlinearity," Journal of Differential Equations, vol. 247, no. 2, pp. 495-510, 2009.

[7] M. Mei, C. Ou, and X.-Q. Zhao, "Global stability of monostable traveling waves for nonlocal time-delayed reaction-diffusion equations," SIAM Journal on Mathematical Analysis, vol. 42, no. 6, pp. 2762-2790, 2010.

[8] J. Wu, D. Wei, and M. Mei, "Analysis on the critical speed of traveling waves," Applied Mathematics Letters, vol. 20, no. 6, pp. 712-718, 2007.

[9] Z.-C. Wang, W.-T. Li, and S. Ruan, "Travelling wave fronts in reaction-diffusion systems with spatio-temporal delays," Journal of Differential Equations, vol. 222, no. 1, pp. 185-232, 2006.

[10] D. D. Joseph and L. Preziosi, "Heat waves," Reviews of Modern Physics, vol. 61, no. 1, pp. 41-73, 1989. 
[11] S. Fedotov, "Traveling waves in a reaction-diffusion system: diffusion with finite velocity and Kolmogorov-PetrovskiiPiskunov kinetics," Physical Review E, vol. 58, no. 4, pp. 51435145, 1998.

[12] F. Andreu, V. Caselles, and J. M. Mazón, "A Fisher-Kolmogorov equation with finite speed of propagation," Journal of Differential Equations, vol. 248, no. 10, pp. 2528-2561, 2010.

[13] J. R. Branco, J. A. Ferreira, and P. da Silva, "Non-Fickian delay reaction-diffusion equations: theoretical and numerical study," Applied Numerical Mathematics, vol. 60, no. 5, pp. 531-549, 2010.

[14] A. Araújo, J. R. Branco, and J. A. Ferreira, "On the stability of a class of splitting methods for integro-differential equations," Applied Numerical Mathematics, vol. 59, no. 3-4, pp. 436-453, 2009.

[15] J. R. Branco, J. A. Ferreira, and P. de Oliveira, "Numerical methods for the generalized Fisher-Kolmogorov-PetrovskiiPiskunov equation," Applied Numerical Mathematics, vol. 57, no. 1, pp. 89-102, 2007.

[16] J.-C. Chang, "Local existence for retarded Volterra integrodifferential equations with Hille-Yosida operators," Nonlinear Analysis. Theory, Methods \& Applications, vol. 66, no. 12, pp. 2814-2832, 2007.

[17] A. Araújo, J. A. Ferreira, and P. Oliveira, "Qualitative behavior of numerical traveling solutions for reaction-diffusion equations with memory," Applicable Analysis, vol. 84, no. 12, pp. 1231-1246, 2005.

[18] A. Araújo, J. A. Ferreira, and P. Oliveira, "The effect of memory terms in diffusion phenomena," Journal of Computational Mathematics, vol. 24, no. 1, pp. 91-102, 2006.

[19] C. Zhang and S. Vandewalle, "General linear methods for Volterra integro-differential equations with memory," SIAM Journal on Scientific Computing, vol. 27, no. 6, pp. 2010-2031, 2006.

[20] W. Wang, C. Zhang, and D. Li, "Asymptotic stability of exact and discrete solutions for neutral multidelay-integro-differential equations," Applied Mathematical Modelling, vol. 35, no. 9, pp. 4490-4506, 2011.

[21] W. Wang and C. Zhang, "Preserving stability implicit Euler method for nonlinear Volterra and neutral functional differential equations in Banach space," Numerische Mathematik, vol. 115, no. 3, pp. 451-474, 2010.

[22] W. Wang and C. Zhang, "Analytical and numerical dissipativity for nonlinear generalized pantograph equations," Discrete and Continuous Dynamical Systems A, vol. 29, no. 3, pp. 1245-1260, 2011.

[23] L. Wen, Y. Yu, and W. Wang, "Generalized Halanay inequalities for dissipativity of Volterra functional differential equations," Journal of Mathematical Analysis and Applications, vol. 347, no. 1, pp. 169-178, 2008.

[24] V. Méndez, S. Fedotov, and W. Horsthemke, Reaction-Transport Systems: Mesoscopic Foundations, Fronts, and Spatial Instabilities, Springer Series in Synergetics, 2010.

[25] A. Bellen and M. Zennaro, Numerical Methods for Delay Differential Equations, Oxford University Press, Oxford, 2003.

[26] H. Brunner, "Recent advances in the numerical analysis of Volterra functional differential equations with variable delays," Journal of Computational and Applied Mathematics, vol. 228, no. 2, pp. 524-537, 2009.

[27] H. Brunner, "Current work and open problems in the numerical analysis of Volterra functional equations with vanishing delays," Frontiers of Mathematics in China, vol. 4, no. 1, pp. 3-22, 2009.
[28] A. Yadav, S. Fedotov, V. Méndez, and W. Horsthemke, "Propagating fronts in reaction C- transport systems with memory," Physics Letters A, vol. 371, pp. 374-378, 2007.

[29] S. Fedotov, "Non-Markovian random walks and nonlinear reactions: subdiffusion and propagating fronts," Physical Review E, vol. 81, no. 1, Article ID 011117, 7 pages, 2010.

[30] S. Fedotov, A. Iomin, and L. Ryashko, "Non-Markovian models for migration-proliferation dichotomy of cancer cells: anomalous switching and spreading rate," Physical Review E, vol. 84, no. 6, Article ID 061131, 8 pages, 2011.

[31] C. Zhang and S. Zhou, "The asymptotic stability of theoretical and numerical solutions for systems of neutral multidelaydifferential equations," Science in China A, vol. 41, no. 11, pp. 1151-1157, 1998.

[32] C.-J. Zhang and S.-F. Li, "Dissipativity and exponentially asymptotic stability of the solutions for nonlinear neutral functional-differential equations," Applied Mathematics and Computation, vol. 119, no. 1, pp. 109-115, 2001.

[33] D. Li, C. Zhang, and W. Wang, "Long time behavior of nonFickian delay reaction-diffusion equations," Nonlinear Analysis. Real World Applications, vol. 13, no. 3, pp. 1401-1415, 2012.

[34] J. R. Cannon and Y. P. Lin, "A priori $L^{2}$ error estimates for finite-element methods for nonlinear diffusion equations with memory,' SIAM Journal on Numerical Analysis, vol. 27, no. 3, pp. 595-607, 1990.

[35] J. R. Cannon and Y. Lint, "Error estimates for semidiscrete finite element methods for parabolic integro-Differential equations," Mathematics of Computation, vol. 187, no. 53, pp. 121-139, 1989.

[36] H. Tian, "Numerical and analytic dissipativity of the $\theta$-method for delay differential equations with a bounded variable lag," International Journal of Bifurcation and Chaos in Applied Sciences and Engineering, vol. 14, no. 5, pp. 1839-1845, 2004. 


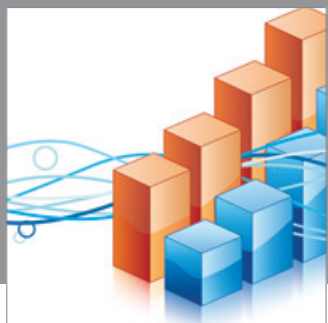

Advances in

Operations Research

mansans

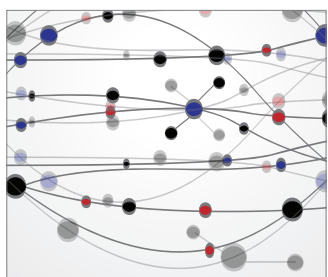

The Scientific World Journal
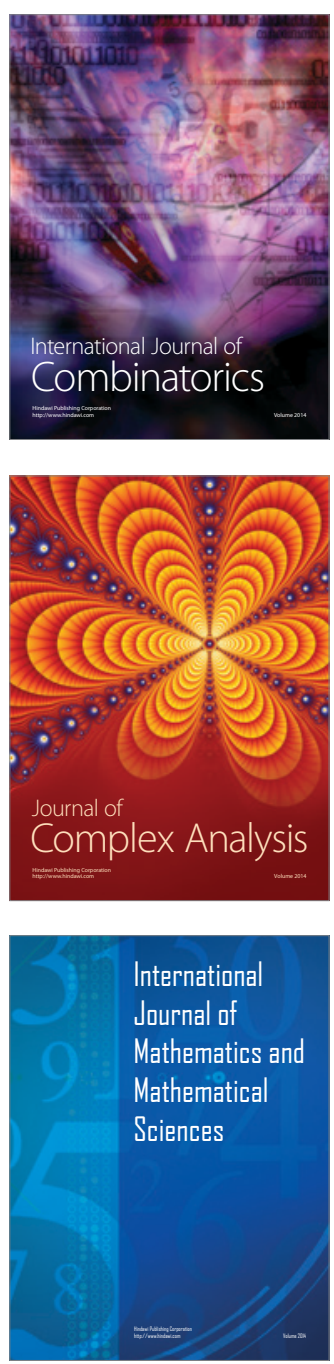
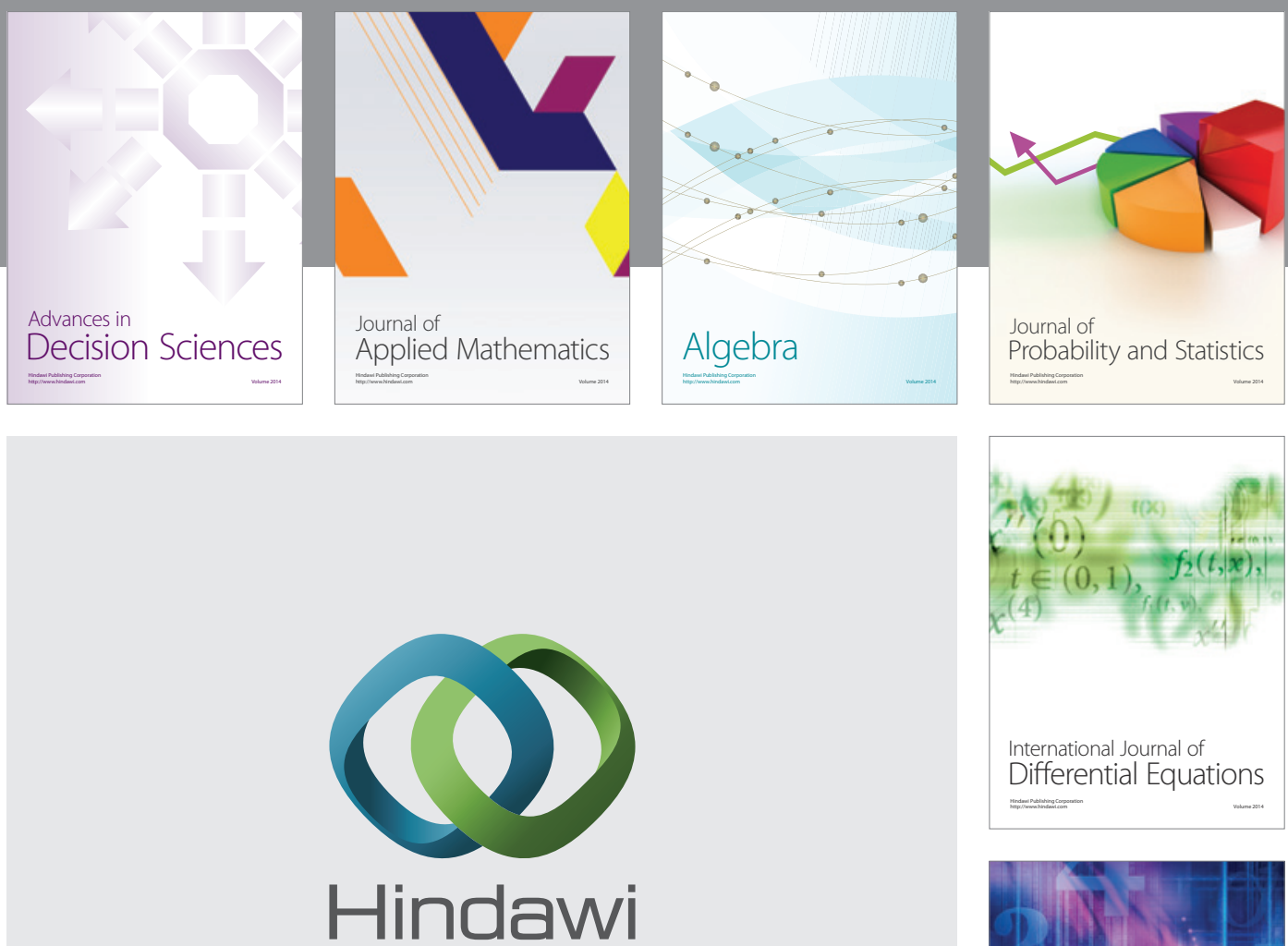

Submit your manuscripts at http://www.hindawi.com
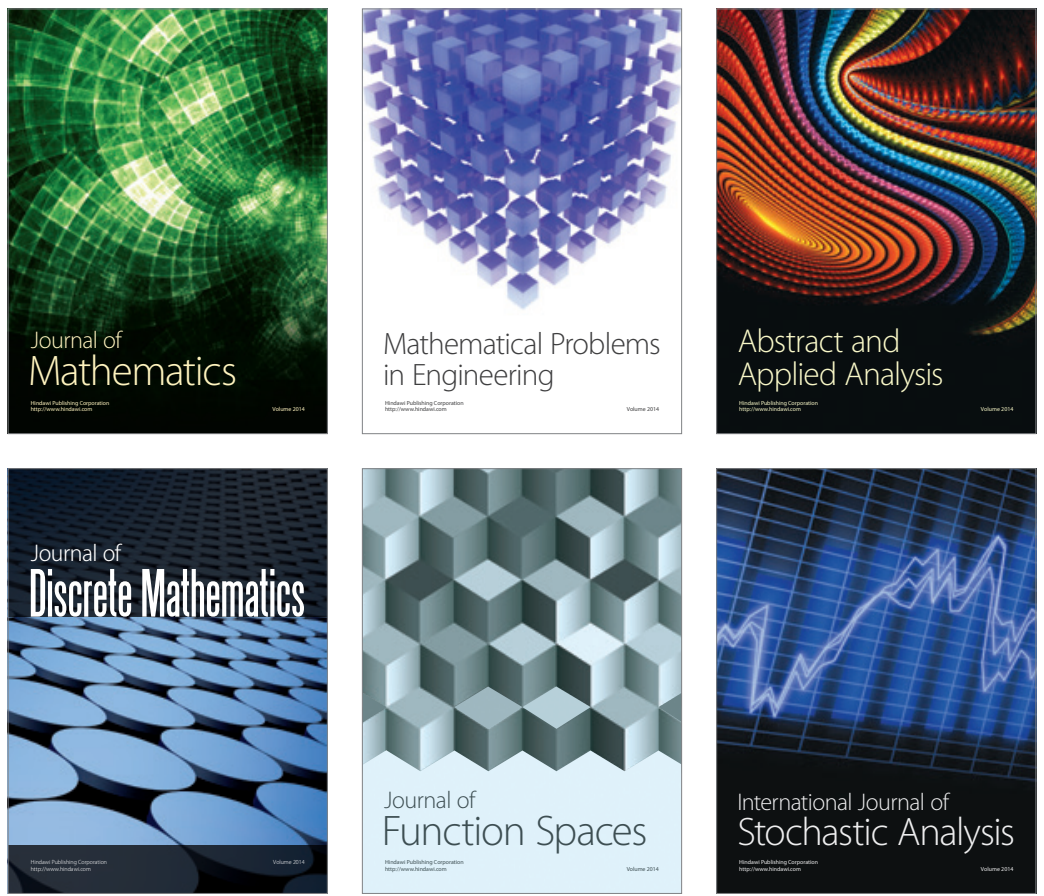

Journal of

Function Spaces

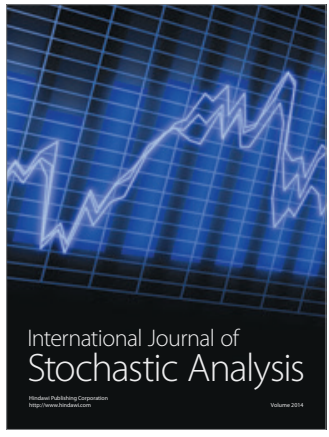

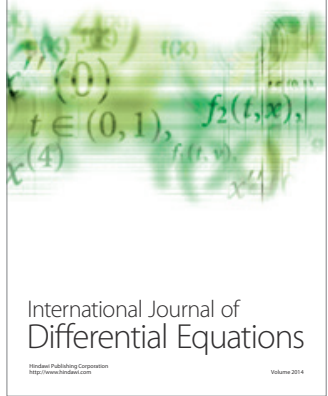
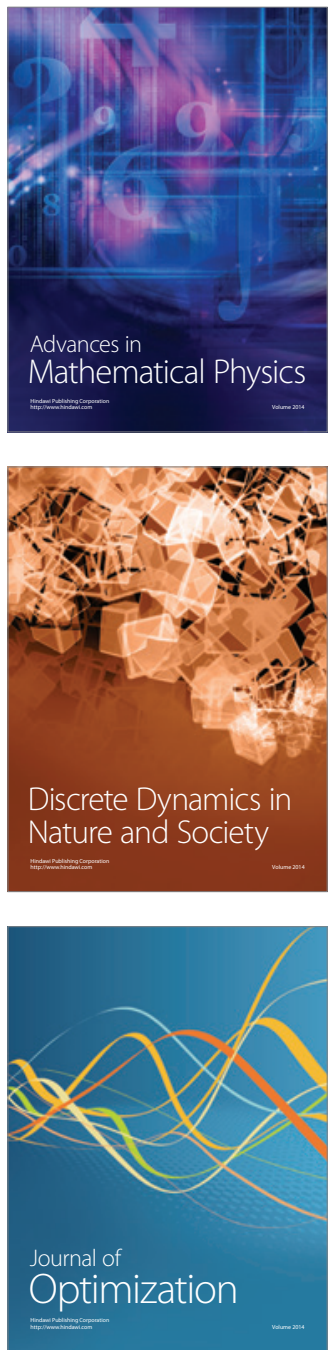